\title{
Ethical challenges in conducting research in humanitarian crisis situations
}

\author{
1. Centre for Bioethics in Eastern and Southern Africa, College of \\ Medicine, Malawi \\ 2. University of North Carolina, Chapel Hill, North Carolina, USA.
}

Joseph Mfutso-Bengo ${ }^{1}$, Francis Masiye ${ }^{1}$, Adamson Muula ${ }^{1,2}$

Corresponding author: Professor J. M. Mfutso-Bengo, Centre for Bioethics in Eastern and Southern Africa (CEBESA), Department of Community Health, College of Medicine, Private Bag 360, Chichiri, Blantyre 3, Malawi Phone: +2651871911 ext 310, Email: mfutsobengo@medcol.mw

\begin{abstract}
Research is vital to accurately describe phenomena in humanitarian emergency situations and to evaluate the effectiveness and appropriateness of interventions. Although the ethical principles of justice, beneficence and respect for autonomy/ respect for persons should be upheld in research, their application in emergency situations may differ from non-emergency situations. Just like in non-emergency situations, research in emergency situations should be conducted in the best interest of the victims or future victims. The research should not unnecessarily expose human subjects and the researcher to careless harm, and should be of adequate scientific rigor. Victims of emergency situations are vulnerable populations that need special protection from exploitation. Technical competency to conduct research in emergency situations should include the ability to conduct a fair risk-benefit assessment in order to come up with a risk management plan, and being culturally sensitive to the needs of the victims of the humanitarian crisis. In emergency situations, the roles of Institutional Review Boards (IRBs) may have to be modified without compromising the ethical standards that health researchers have globally attempted to achieve.
\end{abstract}

\section{Introduction}

Natural and unnatural (man-made disasters) are increasingly being reported across the globe. Research in emergencies is important in order to describe the living situations, assess the needs of the victims of the emergency situations, and to monitor and evaluate interventions so as to provide adequate and safe care to victims of emergency situations. What may be acceptable in the non-emergency situation may have to be adapted and evaluated in humanitarian emergency conditions. ${ }^{1}$ A humanitarian emergency is a crisis which requires immediate attention and response. While the general principles of research ethics used in non-emergency situations are similar to those in emergency situations, that is, adherence to the principles of respect for persons, beneficence and justice, the unique circumstances of emergency situation avail themselves with special considerations as the social fabric may be significantly disrupted and the functioning of institutional review boards (IRBs), where they may have existed, may be compromised. Emergencies may also be associated with perpetrators of violence, who may be the cause of the humanitarian crisis and victims of the ensuing violence, a situation that may not be as applicable to nonemergency situations. Research aspects of a humanitarian crisis can be conducted during or after the emergency situation. In this paper, we attempt to discuss the ethics of conducting research in emergency situations and suggest some guidelines that would enable researchers to have sign posts in ensuring that research conducted in humanitarian crises is of acceptable ethical standard. For the purposes of this paper, an emergency situation and humanitarian crisis have been used interchangeably.

\section{Beneficence}

The guiding principles of research are essentially the same whether in emergency situations or in non-emergency situations. However, research conducted in emergency situations must be in the best interest of victims of the humanitarian crises or future victims. As it is generally agreed that the primary needs of victims of a humanitarian crisis are life, safety and security, these needs should receive the utmost priority. Good intentions for conducting the research should not only be implied but as much as possible they should be demonstrable. The results of any research conducted in humanitarian crisis should benefit the victims directly by improving their life, safety and security unlike in non-emergency situations, where the results of the research may indirectly benefit the research participants but instead provide direct future benefit to the society at large.

\section{Respect for autonomy/respect for persons}

Generally, respect for personal autonomy is provided for when informed consent is obtained from research participants or their legal representatives. This stems from the understanding that individuals are independent moral agents and that those deemed unable to exercise such autonomy are protected and are treated as if they would have made informed choices. Victims of humanitarian crises are a vulnerable group and therefore are worthy of added protection. However, such protection should not be to the extent that they are prevented from participating in research in which they have volunteered to participate. A dilemma exists when the situation they are in provides them an inducement to participate in research. For instance, a hungry victim of a crisis may volunteer more readily to participate in a nutritional supplementation intervention program than if they were not starving. Although where practicable, individual informed consent should be sought, certain situations in an emergency situation may mean that such is not always possible. The consent of the appropriate legal state or community representatives may be sought.

\section{Justice}

The principle of justice is usually manifested in the fair selection of research subjects. Non-beneficial (futile) research should never be carried out in emergency situations. In clinical trials, it is generally agreed that study participants should have the opportunity of accessing the care that is superior when the results of the trial show that one intervention is better than the other. Of course, this notion is modified to suggest that the goal should really be access to the minimum standard of care within that community. Consider a famine where a food supplement such as the ready-to-use food (RTUF) is being tried in an emergency situation. RTUF have 
been reported as beneficial in reducing malnutrition and improving catch-up growth amongst children in Malawi. ${ }^{2,3}$ It may be argued that if found to be more beneficial than some other standard care practices, the study participants should have the benefit of accessing the RTUF from the research team. Now, the challenge that could be anticipated is that those enrolled in the study may leave a proportion of the community suffering from the humanitarian hunger crisis. Tension may araise if the researchers provide RTUF only to the research participants, other persons affected by the hunger, may face harm.

\section{Cultural sensitivity}

Being culturally sensitive is an attribute that research teams needs to exercise. This is due to a number of reasons but mostly to ensure active community involvement as the community does not perceive overt threats to their way of life. For instance, in some cultures, it may not be perceived as appropriate for social public body contact between members of the opposite sex. In some of these cultures, a hand shake between a male and a female, who may not be married to each other, could be perceived inappropriate and a manifestation of sexual advancement. A researcher from another culture who may not be conversant with such cultural norms and values may endanger themselves by such "inappropriate" behavior but could also result in the research work being hampered as the community may not be cooperative as research participants. It is impossible for researchers from another social environment to know all the acceptable and unacceptable cultural practices in another area. It helps however to attempt to be appraised on such cultural practices through either review of the literature or discussions with members of the target community. As much as is practicable, researchers should also attempt to incorporate within their research teams individuals with similar cultural background as the research participants.

A research team that plans to conduct research in any area where contact with human is scheduled should always ensure that there is appropriate recognition or appreciation of the cultural and religious beliefs of the community. In many emergency situations, there may be not only one but multiple cultures and religious beliefs that the researchers may find. In particular areas where emergencies result from cultural and ethnic conflict, involvement of persons within the research team who may be perceived as sympathizers to the perpetrators of the conflict may pose as a challenge and possibly be counter-productive and potentially expose the research team to unnecessary and potentially avoidable harm.

Where the researcher or his/her assistants are not speakers of the language of the victims of the humanitarian crisis, administering a research questionnaire, for instance, poses particular challenges. The interpreters, guides and assistants must be those that will be acceptable to the subjects and the community. A reasonable understanding of the culture is particularly useful. For example, it is probably safe to have women research assistants interviewing fellow women and men interviewing males. Exceptions may occur but as a general rule this may be acceptable. There may be times when it may be possible to get a particular gender (most likely women) to be research assistants in humanitarian crises as women are in many cases perceived not as much threats in many societies. Like in all cases, exceptions do occur.
For instance, involving women as research assistants could potentially expose them to hazards such as sexual abuse in war torn environments.

\section{Non-maleficence}

Theidea of non-maleficence is even more criticalin emergency situations where the researcher is dealing with vulnerable populations. Harm can be avoidable or unavoidable, and anticipated or unanticipated. In conducting research, avoidable and anticipated harm should be minimized. Harm should be prevented for the research team as well as the victims of the humanitarian crisis.

\section{Undue inducement or necessary care}

Just as in non-emergency situations, study participants should never be unduly induced to participate in research. Although study participants in emergency situations are vulnerable, they must participate voluntarily and no inducements should be offered to participants to make them participate in studies. However, life-saving care to victims of emergency situations should never be withheld in order to provide the environment for research and neither should it be withheld to ensure that there is no accusation of inducements. For instance, it is unethical to withhold food intervention just because the researchers later want to study the effectiveness of RTUF i.e. the conditions of human suffering should never be created, aggravated or maintained by the researchers. However, the researchers need to make it clear to the potential subjects that being a beneficiary of an intervention does not have any bearing on the victims' participation in the research, if the intervention itself is not a matter of research. In conducting research, investigators should also consider, anticipate and attempt to prevent misconceptions, misestimation and unwarranted optimism about the intervention ${ }^{4}$. The above terms are thus reported by Horng and Grady:

a) Therapeutic misconception: This term was first described by Appelbaum et al in $1987 .{ }^{5}$ This is where the research participant confuses, or does not differentiate between or "conflates" research and clinical practice.

\section{b) Therapeutic misestimation: This is where the research} participant underestimates risk, or overestimates benefit, or both.

c) Therapeutic optimism: This is where the research participant hopes for the best individual or personal outcome.

In virtually all emergency situations, the primary needs of the victims are preservation of life, safety and security. Even though research may contribute to the realization of these needs, such may not be perceived as such by the victims. The need therefore of truthfulness is particularly crucial in research situations where the subjects may be misled to think that the collection of data is such that the researcher is identifying a particular group i.e. either as beneficiary of impending relief interventions or individuals whose names are to be presented to the aggressors.

\section{Relationship with relief agencies}

In a humanitarian crisis, the researcher should ensure that they work with the humanitarian or relief agencies that are attending to the needs of the victims in terms of safety, security, food, health care and rehabilitation. The researchers should endeavour to limit how much the research activities hamper relief efforts. The basic survival needs of victims should rank first before research pursuit. There may however be circumstances where research may have to precede 
humanitarian relief efforts especially when life is not in immediate danger. Researchers and relief agencies should work in cooperation and desist from creating situations where the efforts of the other may be undermined.

\section{Access to emergency care}

Conducting research in emergency situations will almost inevitably lead the researchers to come in contact with people who may need health and other essential care not available at the time or place where the contact with research subjects is occurring. This raises the question as to what would be the role of researchers in such situations. For instance, there is an individual who has been bitten by a venomous snake as he participated in the study. What would the research team have to do? Immediate health care may not be available within the locality but may be so available elsewhere. It is prudent for the research team to assist as may be practicable within the prevailing resources. Where possible, the research may have to assist in facilitating access to life-saving care.

\section{Scientific rigor}

Just because research is being carried out in emergency situations does not mean that haphazard and unsound research techniques should be employed. If research would lack scientific rigor, that would essentially expose both the research team and subjects to unwarranted cost in time, resources and the sometimes inadvertent hazards that may be inherent in emergency situations. Within the limits that are inherent in an emergency situation, all that would ensure implementation of sound research methodology must be done. There is no room for mediocrity.

While research rigor is a necessity, there must also be demonstrable research value of the study. A study can be of high methodological standard and yet be wanting in how it adds to the body of knowledge that may be useful to enhance standard of life for humanity.

\section{Risk-Benefit assessment}

By its nature, research in emergency situations could potentially expose study participants and the researchers to relatively higher potential for harm than may be the case in research conducted in non-emergency situations. Researchers in emergency situations should weigh the risks as opposed to the potential benefits of such research. Risk can be to the research team itself, research participants or others not directly involved in the research, such as the community. Consider, for instance, a refugee situation which has been created by tribal or ethnic conflict. Research that has potential to alleviate the suffering of the refugees ( for example, the use of RTUF) may be perceived as harmful to the adversary as the research has potential to limit the harm that had been created by the violence. The adversary may then attack the refugee camps or the researchers so that the suffering of the refugees is prolonged and exacerbated. Research should only be conducted where the potential benefits outweigh the potential harms. Anything less than that is unethical.

Having conducted a risk-benefit assessment, and the decision to carry out the research is reached, there should also be a risk management plan in place for both the research team and the study subjects. Negligence defined as absence of due care, can be exhibited through exposure of either the research team or subjects to unnecessary harm without risk management plan in place. While harm may be intended, it must be reasonable to be acceptable but not careless.

\section{Sustainability of potential interventions}

In conducting research in emergency situations, the researchers should give due consideration of the acceptability and sustainability of the intervention in the communities. It is questionable when research introduces an intervention which the communities may not be able to continue accessing or using once the research project has concluded. Researchers should also potentially and overt controversial intervention. An example could be the introduction of genetically modified (GM) food as therapy for malnutrition in a humanitarian crisis. While there are significant benefits to be accrued from such GM crops, the sensitivities and opposition to such technology can not be easily neglected.

\section{Relationships with authorities}

An appropriate relationship with authorities, however organized or legitimate such may be, is an important consideration of conducting research in emergency situations. Authorities may be useful in providing relevant permission to conduct the study, and may provide security for both researchers and subjects during the research period. There are however associated risks that people may be exposed to, for instance by perceptions of being collaborators to authorities. For instance, in situations where the authorities are perceived as aggressors or perpetrators of a social harm, research subject may associate the research team as an equally unwelcome entity. Association with authorities therefore has to be purely on a professional level and this should be defined. Where there is potential for confusion, the research team should endeavour to clear the suspicion through processes that may be in their power, without putting themselves in unnecessary harm.

\section{Exemptions from IRB review}

In some sense, Institutional Review Boards (IRB) can be like bureaucracy. Mattox has suggested that "bureaucracy breeds inaction". 6 The conditions in emergency situations may be such that obtaining IRB approval may not be practicable; for instance, where an IRB does not exist or the urgency of the situation will render the delay by any IRB to be unacceptable, the guiding principle should be the best interest of the victims of the emergency situation. Where such research enhances local capacity to respond, facilitates delivery of appropriate intervention and does not expose subjects and researchers to undue harm, such research may be permissible.

Even in non-emergency situations, some research may be exempted from stringent reviews of Institutional Reviews Boards. Several situations in educational research are exempt from IRB review. These include; research performed on regular or established educational settings and involving practices that are commonly accepted; research on educational tests or observation of public behavior where the subjects can not be later identified or on elected or appointed public officials and research that utilizes existing data which are publicly available or the subjects cannot be identified. Research that is conducted to study or evaluate or examine public benefit of a welfare program for instance, may be IRB exempt when the head of the particular agency or department involved has given permission. 
In emergency situations, research may have to be expedited. For instance, it may be important to study the living situations of the immediate aftermath of a disaster situation, such as the Asian Tsunami disaster. If the formal IRB situation were to be followed, in many cases, by the time such authority was provided, the situation may have changed either due to lapse of time or interventions that may have been implemented. Of course, a retrospective study could be conducted, but depending on the nature of the study, this may not be considered as appropriate. In such situations, IRB approval may not be practical. However, research should endeavour to obtain permission for the recognized authority; which may be the State, local government authority, UN agencies or other similar organizations.

In situations where certain humanitarian crises can be anticipated, it is possible to have IRB review of a research project in advance. Although this may be perceived as "wishing" human suffering, it has to be also appreciated that the researchers do not have a hand in the causation of the suffering but are attempting to contribute to the solution of such disasters.

\section{Especially vulnerable persons}

Although in humanitarian crises large groups of persons experience suffering, the situations of particular groups such as aliens, the elderly, widows, orphans, physically and mentally challenged possibly deserve particular attention as research subjects. These groups are not capable of making individual decisions whether to participate in a study or not. The explicit permission of the relevant authority figures on their behalf may be required. However, this should not override individual choices except in the case of the mentally challenged whose cognitive faculties may be impaired by illness.

\section{Dissemination of findings}

Emergency situations present particular demands with regard to the publication of research findings. The questions as to when, how, to who are particularly pertinent as careless dissemination of findings may predispose both the researchers and the subjects to potential harm. For instance, researchers may identify particular institution's shortfalls in the response to human needs or identify the perpetrators on a social ill. Understandably such findings may not be acceptable by some sections of the community who may feel the researchers have not been a fair representation of their role in the crisis. For instance, a government that has been identified as doing little to alleviate human suffering may penalize, even banning relief agencies from the country of the researcher(s) accusing them of "spying" or intentionally aiming "to tarnish the image of a democratic government".

\section{Summary guidelines}

Although the Code of Ethics for Emergency Nurses ${ }^{7}$ was primarily formulated to deal with clinical emergencies, we find the same to be reasonably appropriate to humanitarian crises. The following are the summary guidelines adapted from the Code:

1. The researcher should ensure that he/she conducts research with compassion and respect for individuals and communities.

2. The researcher should maintain the highest sense of scientific rigor and competency.
3. The researcher should ensure that victims of emergency situation's welfare are not further jeopardized because of the research.

4. The rights to privacy and confidentiality of subjects have to be respected.

5. Research should not hamper relief efforts but rather facilitate the delivery of the same

6. Where appropriate, collaborations with other researchers and agencies should be encouraged in order to enhance care.

7. Research in emergency situations should be carried out only when similar data can not be obtained otherwise.

8. Research should be responsive to the needs of the victims of the emergency.

9. Only research that does not hinder or obstruct effective and appropriate interventions should be carried out.

10. Research should improve interventions and/or local capacity to respond to the current or future emergency.

\section{Conclusion}

Ethical considerations and research guidelines in humanitarian crisis situations should not be, nor be perceived as attempting to limit well-thought out and appropriate research but rather as a tool and process that allow the enhancement of care and protection of vulnerable groups. The ethical principles of beneficence, justice and respect for persons are as applicable as in non-emergency situations. It is important that although this paper has dwelt significantly on research involving human subjects, the guidelines and applicability of the principles may be modified as appropriate when no human subjects are employed as participants. Significant challenges are likely to be experienced in much vulnerable groups such as HIV/AIDS patients, psychiatric patients, orphans and the elderly without families.

\section{Acknowledgements}

The authors of this paper are supported by the European and Developing Countries Clinical Trials Partnership (EDCTP) and the National Institutes of Health-Fogarty International Centre.

\section{References}

1. Leyennar J. (2004). Human immuno-deficiency virus and infant feeding in complex humanitarian emergencies: priorities and policy considerations. Disasters. 2004; 28, 1-15

2. Sandige H., Ndekha M, Briend A, Ashorn P, Manary MJ. Home-based treatment of malnourished Malawian children with locally produced or imported ready-to-use food. J Pediatr Gastroenterol Nutr. 2004; 39: 141-6.

3. Manary MJ, Ndekha M, Ashorn P, Maleta K, Briend A. Home based therapy for severe malnutrition wit ready-to-use food. Arch Dis Child. 2004; 89: 557-61

4. Horng S, Grady S. Misunderstanding in Clinical Care: Distinguishing Therapeutic Misconception, Therapeutic Misestimation, and Therapeutic Optimism. IRB: Ethics and Human Research. 2003; 25(1): 11-16

5. Appelbaum PS, Roth LH, Lidz CW, Benson P, Winslade W. False hopes and best data: Consent to research and the therapeutic misconception. Hastings Center Report. 1987: 12(2): 20-24.

6. Mattox K. The World Trade Center Attack. Disaster Preparedness: health care is ready, but is the bureaucracy? Critical Care. 2001; 5(6), 323-5

7. Emergency Nurses Association. Code of Ethics for Emergency Nurses. 2004 Accessed from: www.emergency.net/ethics.htm 